\title{
Presumed early ovarian cancer with isolated tumor cells in para-aortic sentinel nodes
}

\author{
Presenter: \\ Stefano Uccella \\ Department of Woman and Child Health, Fondazione Policlinico Universitario A. Gemelli, IRCCS, \\ Rome, Italy \\ Presenter: \\ Anna Fagotti \\ Department of Woman and Child Health, Fondazione Policlinico Universitario A. Gemelli, IRCCS, \\ Università Cattolica del Sacro Cuore, Rome, Italy \\ Pathologist: \\ Gian Franco Zannoni \\ Department of Gynecologic Pathology, Fondazione Policlinico Universitario A. Gemelli, IRCCS, \\ Università Cattolica del Sacro Cuore, Rome, Italy \\ Discussant: \\ Robert L Coleman \\ Department of Gynecologic Oncology and Reproductive Medicine, The University of Texas MD \\ Anderson Cancer Center, Houston, Texas, USA
}

Correspondence to

Dr Stefano Uccella, Department of Woman and Child Health, Fondazione Policlinico Universitario A. Gemelli, IRCCS, Rome 8, 00168, Italy; stefucc@ libero.it

Accepted 9 October 2018 Published Online First 1 January 2019

\section{CASE PRESENTATION}

A 51-year-old $\mathrm{G}_{2} \mathrm{P}_{1}$ woman (body mass index $29 \mathrm{~kg} /$ $\mathrm{m}^{2}$ ) was diagnosed with an incidental adnexal mass at a routine screening gynecologic examination. A pelvic ultrasound was performed and it showed evidence of a $5.3 \mathrm{~cm}$ right ovarian mass with irregular margins and vascularized papillary projections (Figure 1). She was then referred to our institution for management. She reported regular menses and no menopausal symptom. Her past medical history was significant for well-controlled hypothyroidism. Her family history was negative for cancer. On physical examination,

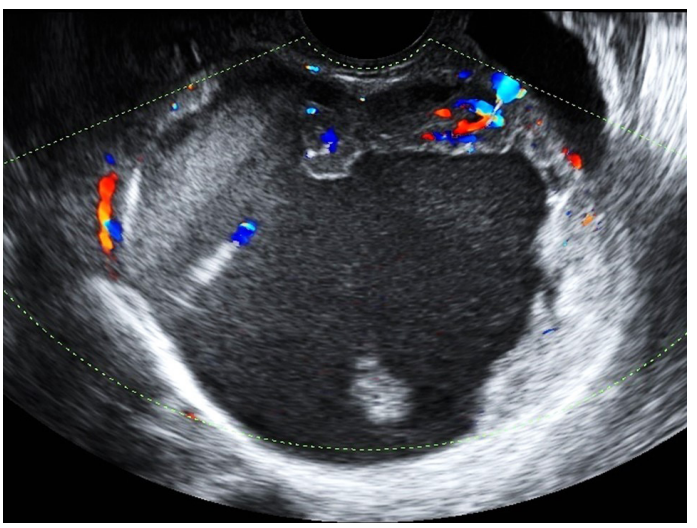

Figure 1 Ultrasonographic image of the right ovarian mass with irregular walls and vascularized projections. the patient was noted to have an approximately $5 \mathrm{~cm}$ non-tender fixed right ovarian mass.

Several serologic markers were obtained and revealed the following: CA125 $=272 \mathrm{UI} / \mathrm{mL}$ (normal range $<35 \mathrm{UI} / \mathrm{mL}$ ), CA19.9=1.44 UI/mL (normal range $<40 \mathrm{UI} / \mathrm{mL}$ ), CA15.3=36.9 UI/mL (normal range $<32.4$ $\mathrm{UI} / \mathrm{mL}$ ), HE4=173 $\mathrm{pmol} / \mathrm{L}$ (normal range $<40 \mathrm{pmol} / \mathrm{L}$ in premenopausal women). An abdomino-pelvic magnetic resonance image confirmed the $5.3 \mathrm{~cm}$ right adnexal mass, with moderately vascularized, irregular intracavitary papillary projections at $\mathrm{T} 1$ - and T2-weighed images. There was also evidence of two uterine fibroids (13 and $15 \mathrm{~mm}$, respectively) and non-specific adenopathy in the right obturator fossa. No carcinomatosis or ascites was described. The chest $X$-ray was negative.

The patient was counseled and consented to undergo laparoscopy for right adnexectomy, frozen section and comprehensive surgical staging in case of ovarian cancer at pathological intra-operative evaluation (she was also offered a hysterectomy given her age and her elevated serum CA125; however, she desired to have an oophorectomy and only to proceed with full hysterectomy and contralateral oophorectomy if the specimen was found to be malignant on frozen section). Before the procedure, the patient consented to participate in the SELLY (SEntinel Lymph node in earLY ovarian cancer) trial. This is a prospective study evaluating the feasibility and accuracy of sentinel node detection in early ovarian cancer. The 
anticipated accrual for this trial is 132 patients. Patients included in this trial have a presumed stage I ovarian cancer and undergo sentinel lymph node mapping using indocyanine green injection in the suspensory and proper ovarian ligament, to identify the sentinel lymph node in the pelvic and lumboaortic areas. A routine systematic lymphadenectomy is then performed (Clinicaltrial.gov registration number: NCT03563781.

At the time of laparoscopy, the right ovary appeared to have an irregular external wall. The contralateral ovary was fixed to the pelvic peritoneum, but no evidence of macroscopic disease was noted. Inspection of the upper abdomen revealed small nodules measuring approximately 1-2 $\mathrm{mm}$ at the level of the right pelvic peritoneum and right diaphragm. A careful and thorough exploration of all the abdominal quadrants, with mobilization of the bowel, was performed, with no other evidence of suspicious disease. After peritoneal washings were obtained, a laparoscopic right salpingo-0ophorectomy was performed. The adnexum was detached from its supporting structures without intra-operative rupture, puncture, or spillage and it was placed intact in an endobag. It was then removed through the umbilical port.

The frozen section evaluation revealed evidence of a high-grade serous adenocarcinoma in the right ovary. All peritoneal nodules were excised en bloc and sent for frozen section and these showed inflammation but no evidence of malignancy.

\section{Dr Coleman}

\section{In addition to performing a contralateral oophorectomy and hysterectomy, is there a role for staging lymphadenectomy given the high-grade nature of the tumor in the right ovary?}

The immediate and somewhat visceral response to this question, if raised intraoperatively, is "yes". Formal staging procedures (peritoneal biopsies, omental resection, nodal evaluation and cytology) in general up-stage $25-30 \%$ of patients, and approximately 1 in 11 women with apparent stage I disease will have lymphatic metastasis as the only site of extraovarian disease. ${ }^{1}$ However, as it relates to the patient, the crux of this question is one of the prognostic and predictive value of lymph node disease status. It is clear in our staging algorithms that such information has prognostic value; that is, patients with nodal metastatic disease have shorter progression-free and overall survival expectations than those who are truly stage I. However, how this information informs a specific patient at a specific point in time is difficult to both grasp and explain accurately, since the individual expectations of success/failure of therapy are binary and an individual's performance is essentially unknown despite the probability estimates in a general population. Thus, while population-based estimates can be given to the patient, the most important information to be gained from nodal status is its therapeutic advantage. In other words, how does the information gained from lymph node evaluation affect treatment recommendations already planned without that information, and does nodal resection incrementally benefit the patient over those who do not have the procedure? In one sense, accurate surgical staging in this case may not measurably change the recommendation for adjuvant therapy, although there are important options discussed below. However, accurate staging that changes an apparent stage I to stage IIIA1(i) could enable participation in clinical trials targeting

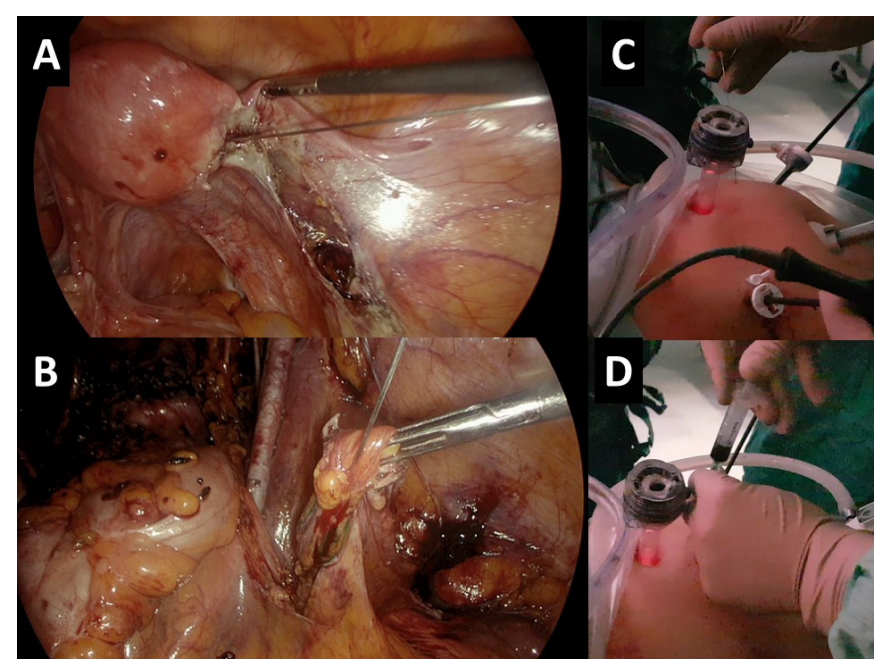

Figure 2 Internal view of the indocyanine green injection in the proper ovarian ligament $(A)$ and suspensory ligament (B) using a 20-gauge needle inserted transcutaneously, under laparoscopic guidance. (C-D) External view of the indocyanine green injection.

'advanced stage' disease. In this latter consideration, accurate staging for this patient would provide more realistic prognostic data and possibly enable clinical trial options, but will not appreciably affect the standard of care therapeutic approach.

At that time, a 20-gauge needle was inserted transcutaneously, under laparoscopic guidance, and $2 \mathrm{~mL}$ of indocyanine green was injected into the right proper ovarian ligament and suspensory ligament, respectively (Figure 2). After approximately $10 \mathrm{~min}$, the retroperitoneal spaces were developed. No sentinel node was detected in the left hemipelvis; however, two right-sided sentinel nodes were identified at the level of the right hemipelvis (in the obturator fossa) and in the inter-aorto-caval region, above the inferior mesenteric artery (Figure 3). Both specimens were detached from the surrounding tissues and sent separately for final pathology with ultrastaging.

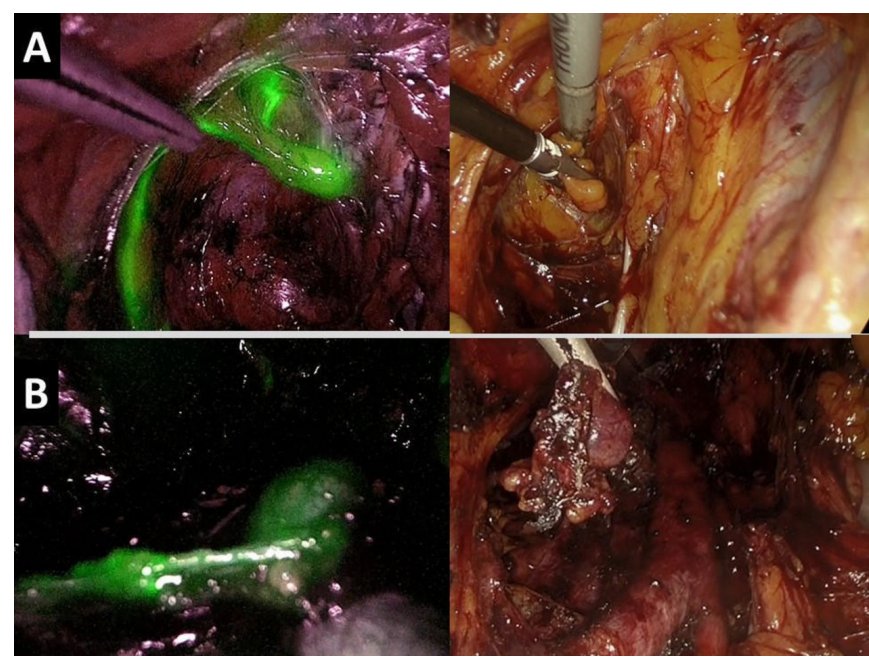

Figure 3 Sentinel nodes in the right obturator fossa $(A)$ and in the inter-cavoaortic region (B). 
Systematic bilateral pelvic and lumbo-aortic Iymphadenectomy was then performed, along with omentectomy, hysterectomy, left salping-0ophorectomy and random peritoneal biopsies.

\section{Dr Coleman}

\section{What is the evidence for consideration of sentinel lymph node mapping in patients with presumed ovarian cancer?}

The concept of sentinel node mapping at any site predicates that non-random lymphatic drainage occurs physiologically, and that a limited number of nodal targets serve as the first or dominant location for that drainage. As such, these sentinel nodes would probabilistically be uniquely informative of nodal spread. Seminal anatomical work by Plentl and Friedman nearly half century ago demonstrated that preferred lymphatic trunks drained the pelvic viscera including the adnexa. ${ }^{2}$ The dominant routes, as expected, are those draining along the ovarian vessels in the paraortic region, as well as the common iliac and rarely presacral trunks. Validation of the concept has been demonstrated in vulval cancer and strong evidence supports similar decision algorithms in cervix and endometrial cancer. However, experience in ovarian cancer is far more limited with recent reports suggesting $<125$ documented cases in the literature and far fewer in patients with apparent stage I cancer. ${ }^{3}$ To date this experience has been favorable with identification of a sentinel lymph node occurring in $>85 \%$ of cases attempted, suggesting the current techniques using dye, radiocolloid and indocyanine green with injections in the para-ovarian fossa are recapitulating anatomical and clinical expectations. However, validation requires performance review in node positive cases and in those in whom apparent limited stage disease is encountered. This is a rare subset but fortunately at least two prospective clinical trials are underway to assess this strategy.

\section{Dr Coleman}

\section{Is there a role for routine frozen section of the sentinel lymph node in order to avoid a systematic lymphadenectomy?}

The answer to this question is more complex than it might seem, as one must consider the circumstances under which the procedure is being done and what the treatment algorithm will be based on the resultant intra-operative data. Since prospective phase III studies assessing the therapeutic role of lymphadenectomy in advanced stage ovarian cancer patients have been negative, the information gathered at the time of frozen section would be essentially the avoidance of routine bilateral staging sampling. However, if adjuvant chemotherapy is already going to be recommended, the information gathered by the procedure will likely add little unless gross disease is appreciated during exploration. Further, since the technique is not validated, the use of intra-operative frozen sectioning would not be necessary since staging sampling would accompany the procedure to assess the performance of sentinel lymph node mapping. If we suppose that sentinel lymph node mapping in ovarian cancer is validated, then the role of routine sentinel lymph node frozen sectioning might be helpful in avoiding lymphadenectomy if a node is identified. Again, the impact of histologically positive or negative findings may have a lesser role. In other tumor

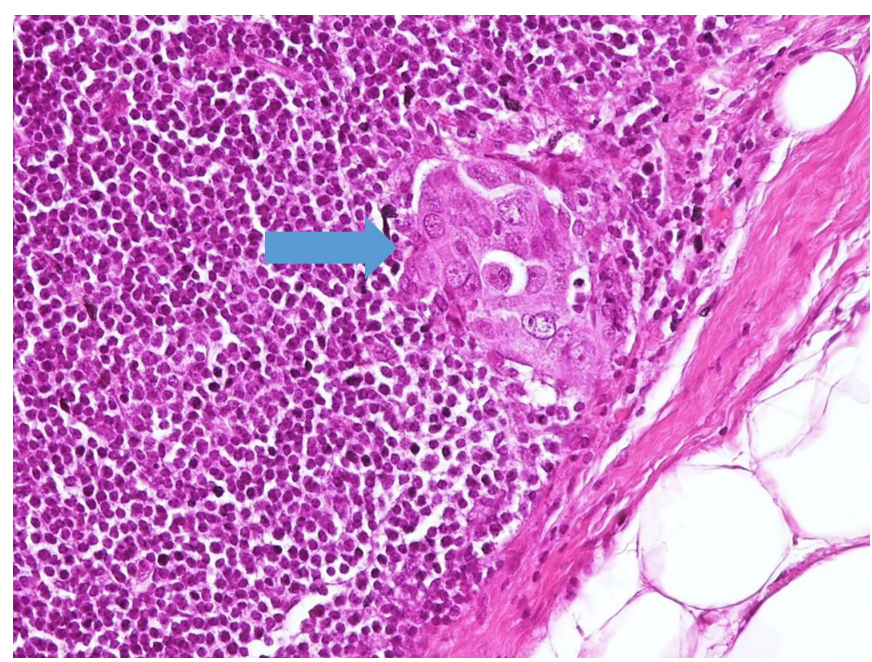

Figure 4 Histological appearance of isolated tumor cells (arrow) in the inter-cavoaortic sentinel node at final pathological examination.

types, including gynecological tumors, the accuracy of intra-operative assessment is high (>90\%), even without step-sectioning and ultrastaging. Further, one-step nucleic acid amplification (CK19 mRNA) has been successful in patients with breast cancer avoiding a delayed secondary operation, and has been piloted in gynecological tumors. ${ }^{4}$ Variance in pathology department expertise and capability for intra-operative assessment makes a recommendation for routine frozen section difficult, even when that information will be used for tailored additional procedures, such as formal lymphadenectomy. In the current case, this would be more difficult to mandate other than to determine if a node was obtained from the mapping procedure.

In our institution, we routinely leave a drain after lymphadenectomy and the patient was noted to have $>500 \mathrm{~mL}$ of serous fluid drainage; thus the patient was discharged on post-operative day 3 after removal of the drain. Final pathology confirmed a high-grade serous adenocarcinoma of the right ovary infiltrating the capsule, with negative peritoneal washing. The contralateral adnexum, uterus, omentum, peritoneal biopsies, and 15 pelvic nodes were negative for disease. The pelvic sentinel lymph nodes were also negative for disease. Interestingly, the specimen labelled 'inter-aortocaval supramesenteric sentinel node' showed no evidence of disease on routine hematoxylin and eosin staining, but the ultrastaging revealed three lymph nodes with isolated tumor cells in two of the three lymph nodes (Figure 4). All other 10 lumbo-aortic nodes were negative for disease.

\section{Dr Zannoni}

The pathological examination of the supramesenteric, inter-cavoaortic node (Figure 4) revealed the presence of isolated tumor cells, defined as fewer than 200 carcinoma cells mainly arranged in small glandular clusters (arrow). Neoplastic cells showed the same morphology as the ovarian mass, consistent with high-grade serous carcinoma. In detail, tumor cells exhibited an abundant eosinophilic cytoplasm and marked nuclear atypia with prominent nucleoli. 


\section{Dr Coleman}

\section{Based on the final pathology, what should be the FIGO stage designation for this patient?}

Although isolated tumor cells are not specifically addressed in the most recent revision of the ovarian cancer FIGO (Fédération Internationale de Gynécologie et d'Obstétrique) staging (published in 2014), a new category in stage III accounts for the more favorable prognosis of women with node-only metastatic disease. Specifically, isolated micrometastatic nodal disease is now subclassified based on the metastatic deposit size $(\leq 10 \mathrm{~mm}$ or $>10 \mathrm{~mm}$ in greatest dimension). However, it is commented that the prognostic implication of this subcategorization is unknown. Under these considerations the patient's FIGO stage would be IIIA1(i).

\section{What is the significance of isolated tumor cells in patients with ovarian cancer?}

The significance of isolated tumor cells in ovarian cancer is largely unknown. However, with systematic ultra-staging by step sectioning combined with metastatic disease cellular marker assessment (eg, cytokeratin immunohistochemistry and CK19 mRNA copy number), the prevalence of micro-metastases is rising. Acknowledgement is also finding its way into the staging system where isolated tumor cells are sometimes designated as pNO(+). Many feel this is justified in breast cancer for instance, as some studies have associated isolated tumor cells with poorer prognosis, higher probability for non-sentinel lymph node metastases, and higher rates of local and distant recurrence. ${ }^{6}$ However, management algorithms in many cases are essentially unchanged despite finding isolated tumor cells, highlighting that the clinical predictive value is low or uncertain. Given this uncertainty, a more global assessment of factors driving clinical care recommendation would be prudent. In ovarian cancer specifically, adjuvant therapy is recommended for nearly all women with invasive disease, so the clinical conundrum would really only apply to those women with grade 1 (or low grade) or grade 2, stage IA or IB disease. In such cases and without data to the contrary, finding isolated tumor cells would likely lead to a more aggressive treatment posture such as the administration of chemotherapy.

\section{Would you recommend adjuvant treatment to this patient based solely on the isolated tumor cells?}

As stated above, conservatively, one would have to consider isolated tumor cells as a reflection of early metastatic disease. Some reports in other solid tumors, such as endometrial, breast cancer and melanoma, have implied that patients with isolated tumor cell-only positive nodes had higher risk factors or fared worse than those who were node negative. ${ }^{7}$ However, as more data emerge, it may be revealed that isolated tumor cells have limited additional independent prognostic implications. ${ }^{9}$ Nevertheless, in the current case, other prognostic factors (high grade) are dictating the treatment recommendation, which is also appropriate for stage III disease. As stated above, the incremental therapeutic benefit of removing these nodes is likely to be trivial.

The patient was counseled to undergo chemotherapy with carboplatin (AUC 5) and paclitaxel $\left(175 \mathrm{mg} / \mathrm{m}^{2}\right)$ every 21 days for six cycles, and at the time of this report she had completed the fourth cycle of such therapy.

\section{Dr Coleman}

\section{How many total cycles of therapy would you recommend for this patient?}

I would agree with the authors' stated plan. Although data from GOG-157 showed that an abbreviated course of combination chemotherapy was statistically the same as six cycles of standard therapy, this patient is considered to be potentially curable and such recommendations would need to be looked at carefully. ${ }^{10}$ it is noteworthy that the estimated rate of recurrence at 5 years for both stage I and stage II patients was higher, albeit not statistically, among patients receiving three cycles of paclitaxel and carboplatin. In a subsequent analysis isolating the high-grade serous subgroup, an improvement in disease-free survival with six cycles of the therapy was suggested, raising the hypothesis that a full course of chemotherapy is needed in these patients. ${ }^{11}$ It is clear from observational (non-treatment) studies previously reported that a substantial portion of these patients do not have a recurrence and as such do not require chemotherapy. However, those who do have a recurrence are usually found with metastatic disease given that there is no diagnostic survey that can identify early or limited recurrent disease. Not surprisingly, these patients had survival characteristics similar to those with advanced stage primary disease (eg, median progression-free survival of 14 months). ${ }^{12}$

\section{DISCUSSANT CLOSING SUMMARY}

The authors are to be commended on their investigation of lymphatic mapping and sentinel node identification in ovarian cancer patients as part of a prospective clinical trial. The finding of metastatic disease in otherwise grossly apparent stage I disease has been known for some time. However, the recommendations for adjuvant therapy have largely been founded on an incomplete understanding of the pathophysiology of disease in these patients. As mentioned, even patients with high-grade limited stage disease, for whom adjuvant therapy has been routinely recommended, are cured by the surgical procedure alone. Techniques for identifying early metastatic disease, such as the sentinel node mapping concept, may help to further define the precision of therapy needed for the patient. Currently, our diagnostic evaluation of these highly selected lymph nodes is limited to immunohistochemistry and molecular survey, but it is likely that much more detailed analysis will become available, including, for instance, how the immune system is educated by the presence of these tumor cells. Through this continued work, highly precise individualized treatment regimens may subsequently emerge.

Provenance and peer review Not commissioned; externally peer reviewed.

\section{REFERENCES}

1. Ayhan A, Gultekin M, Dursun P, et al. Metastatic lymph node number in epithelial ovarian carcinoma: does it have any clinical significance? Gynecol Oncol 2008;108:428-32.

2. Plentl A. Lymphatic system of the female genitalia. Lymphatics of the cervix uteri, ed FE Plentl. 2. Philadelphia: W.B. Saunders Co. 11, 1971.

3. Uccella S, Gisone B, Stevenazzi G, et al. Laparoscopic sentinel node detection with ICG for early ovarian cancer: description of a technique and literature review. Eur J Obstet Gynecol Reprod Biol 2018;221:193-4. 
4. Banerjee SM, Williams NR, Davidson TI, et al. The use of onestep nucleic acid amplification (OSNA) and tumour related factors in the treatment of axillary breast cancer: a predictive model. Eur J Surg Oncol 2016;42:641-9.

5. López-Ruiz ME, Diestro MD, Yébenes L, et al. One-step nucleic acid amplification (OSNA) for the detection of sentinel lymph node metastasis in endometrial cancer. Gynecol Oncol 2016;143:54-9.

6. Patani N, Mokbel K. Clinical significance of sentinel lymph node isolated tumour cells in breast cancer. Breast Cancer Res Treat 2011;127:325-34.

7. Oh TY, Moon SM, Shin US, et al. Impact on prognosis of lymph node micrometastasis and isolated tumor cells in stage II colorectal cancer. J Korean Soc Coloproctol 2011;27:71-7.

8. van Akkooi AC, de Wilt JH, Verhoef $\mathrm{C}$, et al. Isolated tumor cells and long-term prognosis of patients with melanoma. Ann Surg Oncol 2008;15:1547-8.
9. Kim $\mathrm{CH}$, Soslow RA, Park KJ, et al. Pathologic ultrastaging improves micrometastasis detection in sentinel lymph nodes during endometrial cancer staging. Int J Gynecol Cancer 2013;23:964-70.

10. Bell J, Brady MF, Young RC, et al. Randomized phase III trial of three versus six cycles of adjuvant carboplatin and paclitaxel in early stage epithelial ovarian carcinoma: a Gynecologic Oncology Group study. Gynecol Oncol 2006;102:432-9.

11. Chan JK, Tian C, Fleming GF, et al. The potential benefit of 6 vs. 3 cycles of chemotherapy in subsets of women with early-stage high-risk epithelial ovarian cancer: an exploratory analysis of a Gynecologic Oncology Group study. Gynecol Oncol 2010;116:301-6.

12. Kolomainen DF, A'Hern R, Coxon FY, et al. Can patients with relapsed, previously untreated, stage I epithelial ovarian cancer be successfully treated with salvage therapy? J Clin Oncol 2003;21:3113-8. 